\title{
A new explicit triple hierarchical problem over the set of fixed points and generalized mixed equilibrium problems
}

\author{
Thanyarat Jitpeera and Poom Kumam ${ }^{*}$
}

\author{
* Correspondence: poom. \\ kum@kmutt.ac.th \\ Department of Mathematics, \\ Faculty of Science, King Mongkut's \\ University of Technology Thonburi \\ (KMUTT), Bangmod, Thrungkru, \\ Bangkok 10140, Thailand
}

\begin{abstract}
In this article, we introduce and consider the triple hierarchical over the fixed point set of a nonexpansive mapping and the generalized mixed equilibrium problem set of an inverse-strongly monotone napping. The strong convergence of the algorithm is proved under some mild conditions. Our results generalize and improve the results of Marino and $\mathrm{Xu}$ and some authors.

Mathematics Subject Classification (2000): 47H09; 47H10; 47J20; 49J40; 65J15.

Keywords: nonexpansive mapping, strong converge, variational inequality, projection, hierarchical problem, Hilbert space
\end{abstract}

\section{Introduction}

Let $C$ be a closed convex subset of a real Hilbert space $H$ with the inner product $\langle\cdot, \cdot\rangle$ and the norm $\|\cdot\|$. We denote weak convergence and strong convergence by notations $\rightarrow$ and $\rightarrow$, respectively. Let $F$ be a bifunction of $H \times H$ into $\mathcal{R}$, where $\mathcal{R}$ is the set of real numbers. A mapping $A$ be a nonlinear mapping. The generalized mixed equilibrium problem is to find $x \in C$ such that

$$
F(x, y)+\langle A x, y-x\rangle+\varphi(y)-\varphi(x) \geq 0, \quad \forall y \in C
$$

The set of solutions of (1.1) is denoted by $\operatorname{GMEP}(F, \phi, A)$. If $\phi \equiv 0$, the problem (1.1) is reduced into the generalized equilibrium problem is to find $x \in C$ such that

$$
F(x, y)+\langle A x, y-x\rangle \geq 0, \quad \forall y \in C .
$$

The set of solutions of (1.2) is denoted by $\operatorname{GEP}(F, A)$. If $A \equiv 0$, the problem (1.1) is reduced into the mixed equilibrium problem is to find $x \in C$ such that

$$
F(x, y)+\varphi(y)-\varphi(x) \geq 0, \quad \forall y \in C .
$$

The set of solutions of (1.3) is denoted by $\operatorname{MEP}(F, \phi)$. If $A \equiv 0$ and $\phi \equiv 0$, the problem (1.1) is reduced into the equilibrium problem [1] is to find $x \in C$ such that

$$
F(x, y) \geq 0, \quad \forall y \in C
$$


The set of solutions of (1.4) is denoted by $E P(F)$. If $F \equiv 0$ and $\phi \equiv 0$, the problem (1.1) is reduced into the Hartmann-Stampacchia variational inequality [2] is to find $x \in C$ such that

$$
\langle A x, y-x\rangle \geq 0, \quad \forall y \in C .
$$

The set of solutions of (1.5) is denoted by $\operatorname{VI}(C, A)$. The variational inequality has been extensively studied in the literature [3,4]. A mapping $A$ of $C$ into itself is called an $\alpha$-inverse-strongly monotone if there exists a positive real number $\alpha$ such that

$$
\langle A x-A y, x-y\rangle \geq \alpha\|A x-A y\|^{2}, \quad \forall x, y \in C .
$$

A mapping $f: C \rightarrow C$ is called a $\rho$-contraction if there exists a constant $\rho \in[0,1)$ such that

$$
\|f(x)-f(y)\| \leq \rho\|x-y\|, \quad \forall x, y \in C .
$$

A mapping $S: C \rightarrow C$ is called nonexpansive if

$$
\|S x-S y\| \leq\|x-y\|, \quad \forall x, y \in C .
$$

A point $x \in C$ is a fixed point of $S$ provided $S x=x$. Denote by $F(S)$ the set of fixed points of $S$; that is, $F(S)=\{x \in C: S x=x\}$. If $C$ is bounded closed convex and $S$ is a nonexpansive mapping of $C$ into itself, then $F(S)$ is nonempty [5]. Let $A$ and $B$ are two monotone operators, we consider the hierarchical problem over generalized mixed equilibrium problem: Find a point $x^{*} \in \operatorname{GMEP}(F, \phi, B)$ such that

$$
\left\langle A x^{*}, y-x^{*}\right\rangle \geq 0, \quad \forall y \in \operatorname{GMEP}(F, \varphi, B) .
$$

We discuss the hierarchical problem over fixed point: Find a point $x^{*} \in F(S)$ such that

$$
\left\langle A x^{*}, y-x^{*}\right\rangle \geq 0, \quad \forall y \in F(S) .
$$

Yao et al. [6] considered the hierarchical problem over generalize equilibrium problem and the set of fixed point, $x_{s, t}$ be defined implicitly by

$$
x_{s, t}=s\left[t f\left(x_{s, t}\right)+(1-t)\left(x_{s, t}-\lambda A x_{s, t}\right)\right]+(1-s) T_{r}\left(x_{s, t}-r B x_{s, t}\right), \quad s, t \in(0,1),
$$

for each $(s, t) \in(0,1)^{2}$. The net $x_{s, t}$ hierarchically converges to the unique solution $x^{*}$ of the hierarchical problem: Find a point $x^{*} \in \operatorname{GEP}(F, B)$ such that

$$
\left\langle A x^{*}, x-x^{*}\right\rangle \geq 0, \quad \forall x \in \operatorname{GEP}(F, B),
$$

where $A$ and $B$ are two monotone operators. The solution set of (1.9) is denoted by $\Omega$.

Marino and $\mathrm{Xu}$ [7] studied an explicit algorithm, which generated a sequence $\left\{x_{n}\right\}$ recursively by the formula: For the initial guess $x_{0} \in C$ is arbitrary

$$
x_{n+1}=\lambda_{n} f\left(x_{n}\right)+\left(1-\lambda_{n}\right)\left(\alpha_{n} V x_{n}+\left(1-\alpha_{n}\right) T x_{n}\right), \quad \forall n \geq 0,
$$

where $\left\{\alpha_{n}\right\}$ and $\left\{\lambda_{n}\right\}$ are sequences in $(0,1)$ satisfy some conditions. Let $T, V: C \rightarrow C$ are two nonexpansive self mappings and $f$ is a contraction on $C$. Then $\left\{x_{n}\right\}$ converges strongly to a solution, which solves another variational inequality. Recently, Jitpeera and Kumam [8] introduced and studied the iterative algorithm for solving a common 
element of the set of solution of fixed point for a nonexpansive mapping, the set of solution of generalized mixed equilibrium problem, and the set of solution of the variational inclusion. They proved that the sequence converges strongly to a common element of the above three sets under some mild conditions.

In this article, we consider the hierarchical problem over the set of fixed point and generalized mixed equilibrium problem, which contains (1.6) and (1.7): Find a point $x^{*}$ $\in \Xi:=F(S) \cap \operatorname{GMEP}(F, \phi, B)$ such that

$$
\left\langle A x^{*}, x-x^{*}\right\rangle \geq 0, \quad \forall x \in \Xi:=F(S) \cap \operatorname{GMEP}(F, \varphi, B),
$$

where $A$ and $B$ are monotone operators. This solution set of (1.11) is denoted by $\Upsilon$

We present and construct a new iterative algorithm for solving the problem (1.11). The strong convergence for the proposed algorithm to the solution is derived under some assumptions. Our results generalize and improve the results of Marino and Xu [7] and some authors.

\section{Preliminaries}

Let $H$ be a real Hilbert space and $C$ be a nonempty closed convex subset of $H$. Recall that the metric (nearest point) projection $P_{C}$ from $H$ onto $C$ assigns to each $x \in H$, the unique point in $P_{C} x \in C$ satisfying the property

$$
\left\|x-P_{C} x\right\|=\min _{y \in C}\|x-y\| .
$$

The following characterizes the projection $P_{C}$. We recall some lemmas which will be needed in the rest of this article.

Lemma 2.1. The function $x \in C$ is a solution of the variational inequality (1.5) if and only if $x \in C$ satisfies the relation $x=P_{C}(x-\lambda A x)$ for all $\lambda>0$.

Lemma 2.2. For a given $z \in H, u \in C, u=P_{C} z \Leftrightarrow\langle u-z, v-u\rangle \geq 0, \forall v \in C$. It is well known that $P_{C}$ is a firmly nonexpansive mapping of $H$ onto $C$ and satisfies

$$
\left\|P_{C} x-P_{C} y\right\|^{2} \leq\left\langle P_{C} x-P_{C} y, x-y\right\rangle, \quad \forall x, y \in H .
$$

Moreover, $P_{C} x$ is characterized by the following properties: $P_{C} x \in C$ and for all $x \in H$, $y \in C$,

$$
\left\langle x-P_{C} x, y-P_{C} x\right\rangle \leq 0 .
$$

Lemma 2.3. There holds the following inequality in an inner product space $H$

$$
\|x+y\|^{2} \leq\|x\|^{2}+2\langle y, x+y\rangle, \quad \forall x, y \in H .
$$

Lemma 2.4. [9] Let $C$ be a closed convex subset of a real Hilbert space $H$ and let $S: C \rightarrow$ $C$ be a nonexpansive mapping. Then I - S is demiclosed at zero, that is,

$$
x_{n} \rightarrow x \text { and } x_{n}-S x_{n} \rightarrow 0
$$

imply $x=S x$.

For solving the generalized mixed equilibrium problem and the mixed equilibrium problem, let us give the following assumptions for the bifunction $F, \phi$ and the set $C$ :

(A1) $F(x, x)=0$ for all $x \in C$; 
(A2) $F$ is monotone, i.e., $F(x, y)+F(y, x) \leq 0$ for all $x, y \in C$;

(A3) for each $y \in C, x \mapsto F(x, y)$ is weakly upper semicontinuous;

(A4) for each $x \in C, y \mapsto F(x, y)$ is convex;

(A5) for each $x \in C, y \mapsto F(x, y)$ is lower semicontinuous;

(B1) for each $x \in H$ and $r>0$, there exist a bounded subset $D_{x} \subseteq C$ and $y_{x} \in C$ such that for any $z \in C \backslash D_{x}$,

$$
F\left(z, y_{x}\right)+\varphi\left(y_{x}\right)-\varphi(z)+\frac{1}{r}\left\langle y_{x}-z, z-x\right\rangle<0
$$

(B2) $C$ is a bounded set;

(B3) for each $x \in H$ and $r>0$, there exist a bounded subset $D_{x} \subseteq C$ and $y_{x} \in C$ such that for any $z \in C \backslash D_{x}$,

$$
\varphi\left(y_{x}\right)-\varphi(z)+\frac{1}{r}\left\langle y_{x}-z, z-x\right\rangle<0 ;
$$

(B4) for each $x \in H$ and $r>0$, there exist a bounded subset $D_{x} \subseteq C$ and $y_{x} \in C$ such that for any $z \in C \backslash D_{x}$,

$$
F\left(z, y_{x}\right)+\frac{1}{r}\left\langle y_{x}-z, z-x\right\rangle<0 .
$$

Lemma 2.5. [10]Let $C$ be a nonempty closed convex subset of a real Hilbert space $H$. Let $F$ be a bifunction from $C \times C$ to Rsatisfying (A1) - (A5) and let $\varphi: C \rightarrow \mathcal{R}$ be a proper lower semicontinuous and convex function. For $r>0$ and $x \in H$, define a mapping $T_{r}: H \rightarrow C$ as follows.

$$
T_{r}(x)=\left\{z \in C: F(z, y)+\varphi(y)-\varphi(z)+\frac{1}{r}\langle y-z, z-x\rangle \geq 0, \forall y \in C\right\}
$$

for all $x \in H$. Assume that either (B1) or (B2) holds. Then, the following results hold:

(1) For each $x \in H, T_{r}(x) \neq \varnothing$;

(2) $T_{r}$ is single-valued;

(3) $T_{r}$ is firmly nonexpansive, i.e., for any $x, y \in H$, $\left\|T_{r} x-T_{r} y\right\|^{2} \leq\left\langle T_{r} x-T_{r} y, x-y\right\rangle$;

(4) $F\left(T_{r}\right)=\operatorname{MEP}(F, \phi)$;

(5) $\operatorname{MEP}(F, \phi)$ is closed and convex.

Lemma 2.6. [11]Assume $\left\{a_{n}\right\}$ is a sequence of nonnegative real numbers such that

$$
a_{n+1} \leq\left(1-\gamma_{n}\right) a_{n}+\gamma_{n} \delta_{n}+\beta_{n}, \quad \forall n \geq 0,
$$

where $\left\{\gamma_{n}\right\},\left\{\beta_{n}\right\} \subset(0,1)$ and $\left\{\delta_{n}\right\}$ is a sequence in $\mathcal{R}$ such that

(i) $\sum_{n=1}^{\infty} \gamma_{n}=\infty$;

(ii) either $\lim \sup _{n \rightarrow \infty} \delta_{n} \leq 0$ or $\sum_{n=1}^{\infty} \gamma_{n}\left|\delta_{n}\right|<\infty$;

(iii) $\sum_{n=1}^{\infty} \beta_{n}<\infty$.

Then $\lim _{n \rightarrow \infty} a_{n}=0$. 


\section{Strong convergence theorems}

In this section, we introduce an iterative algorithm for solving some the hierarchical problem over the set of fixed point and generalized mixed equilibrium problem.

Theorem 3.1. Let $H$ be a real Hilbert space, $A: C \rightarrow C$ be an $\alpha$-inverse-strongly monotone, $f: C \rightarrow C$ be a $\rho$-contraction with coefficient $\rho \in[0,1)$ and $S, V: C \rightarrow C$ be two nonexpansive mappings. Let $B: C \rightarrow C$ be a $\beta$-inverse-strongly monotone and $F$ be a bifunction from $C \times C \rightarrow \mathcal{R}$ satisfying (A1)-(A5) and let $\varphi: C \rightarrow \mathcal{R}$ is convex and lower semicontinuous with either (B1) or (B2). Assume that $\Xi:=F(S) \cap \operatorname{GMEP}(F, \phi, B)$ is nonempty. Suppose $\left\{x_{n}\right\}$ is a sequence generated by the following algorithm with $x_{0} \in$ $C$ arbitrarily:

$$
x_{n+1}=\beta_{n} f\left(x_{n}\right)+\left(1-\beta_{n}\right)\left[\alpha_{n} V\left(I-\lambda_{n} A\right) x_{n}+\left(1-\alpha_{n}\right) S T_{r_{n}}\left(x_{n}-r_{n} B x_{n}\right)\right],
$$

where $\left\{\alpha_{n}\right\}$ and $\left\{\beta_{n}\right\} \subset(0,1)$ and $\lambda_{n} \in(0,2 \alpha), r_{n} \in(0,2 \beta)$ satisfy the following conditions:

(C1): $\alpha_{n} \lambda_{n}<\alpha_{n}<\gamma \beta_{n}$ for all $n$ and some constant $\gamma$;

(C2): $\lim _{n \rightarrow \infty} \beta_{n}=0, \sum_{n=1}^{\infty} \beta_{n}=\infty, \lim _{n \rightarrow \infty} \frac{\beta_{n-1}}{\beta_{n}}=1$;

(C3): $\lim _{n \rightarrow \infty} \frac{\alpha_{n-1}}{\alpha_{n}}=1$;

(C4): $\sum_{n=1}^{\infty}\left|\lambda_{n}-\lambda_{n-1}\right|<\infty$;

(C5): $\sum_{n=1}^{\infty}\left|r_{n}-r_{n-1}\right|<\infty, \lim \inf _{n \rightarrow \infty} r_{n}>0$.

Then $\left\{x_{n}\right\}$ converges strongly to $x^{*} \in \Upsilon$, which is the unique solution of the variational inequality:

$$
\left\langle(I-f) x^{*}, x-x^{*}\right\rangle \geq 0, \quad \forall x \in \Upsilon .
$$

Proof. We will divide the proof into five steps.

Step 1. We will show $\left\{x_{n}\right\}$ is bounded. Since $A, B$ are $\alpha, \beta$-inverse-strongly monotone mappings, we have

$$
\begin{aligned}
\left\|\left(I-\lambda_{n} A\right) x-\left(I-\lambda_{n} A\right) y\right\|^{2} & =\left\|(x-\gamma)-\lambda_{n}(A x-A y)\right\|^{2} \\
& =\|x-\gamma\|^{2}-2 \lambda_{n}\langle x-\gamma, A x-A y\rangle+\lambda_{n}^{2}\|A x-A y\|^{2} \\
& \leq\|x-y\|^{2}+\lambda_{n}\left(\lambda_{n}-2 \alpha\right)\|A x-A y\|^{2} \\
& \leq\|x-y\|^{2} .
\end{aligned}
$$

By Lemma 2.5, we have $u_{n}=T_{r_{n}}\left(x_{n}-r_{n} B x_{n}\right)$ for all $n \geq 0$. Then, we have

$$
\begin{aligned}
\left\|u_{n}-q\right\|^{2} & \leq\left\|T_{r_{n}}\left(x_{n}-r_{n} B x_{n}\right)-T_{r_{n}}\left(q-r_{n} B q\right)\right\|^{2} \\
& \leq\left\|\left(x_{n}-r_{n} B x_{n}\right)-\left(q-r_{n} B q\right)\right\|^{2} \\
& \leq\left\|x_{n}-q\right\|^{2}+r_{n}\left(r_{n}-2 \beta\right)\left\|B x_{n}-B q\right\|^{2} \\
& \leq\left\|x_{n}-q\right\|^{2} .
\end{aligned}
$$


For any $q \in \Xi$. Since $V, I-\lambda_{n} A$ and $T_{r_{n}}$ are nonexpansive mappings, we have

$$
\begin{aligned}
\left\|x_{n+1}-q\right\| & =\left\|\beta_{n} f\left(x_{n}\right)+\left(1-\beta_{n}\right)\left[\alpha_{n} V\left(I-\lambda_{n} A\right) x_{n}+\left(1-\alpha_{n}\right) S T_{r_{n}}\left(x_{n}-r_{n} B x_{n}\right)\right]-q\right\| \\
& \leq \beta_{n}\left\|f\left(x_{n}\right)-q\right\|+\left(1-\beta_{n}\right)\left\|\alpha_{n} V\left(I-\lambda_{n} A\right) x_{n}+\left(1-\alpha_{n}\right) S T_{r_{n}}\left(x_{n}-r_{n} B x_{n}\right)-q\right\| \\
& \leq \beta_{n}\left\|f\left(x_{n}\right)-f(q)\right\|+\beta_{n}\|f(q)-q\|+\left(1-\beta_{n}\right) \alpha_{n}\left\|V\left(I-\lambda_{n} A\right) x_{n}-q\right\| \\
& +\left(1-\beta_{n}\right)\left(1-\alpha_{n}\right)\left\|S T_{r_{n}}\left(x_{n}-r_{n} B x_{n}\right)-q\right\| \\
& \leq \beta_{n} \rho\left\|x_{n}-q\right\|+\beta_{n}\|f(q)-q\|+\left(1-\beta_{n}\right) \alpha_{n}\left(\left\|V\left(I-\lambda_{n} A\right) x_{n}-V\left(I-\lambda_{n} A\right) q\right\|\right. \\
& \left.+\left\|V\left(I-\lambda_{n} A\right) q-q\right\|\right)+\left(1-\beta_{n}\right)\left(1-\alpha_{n}\right)\left\|T_{r_{n}}\left(x_{n}-r_{n} B x_{n}\right)-q\right\| \\
& \leq \beta_{n} \rho\left\|x_{n}-q\right\|+\beta_{n}\|f(q)-q\|+\left(1-\beta_{n}\right) \alpha_{n}\left(\left\|x_{n}-q\right\|+\|V q-q\|+\lambda_{n}\|V A q\|\right) \\
& +\left(1-\beta_{n}\right)\left(1-\alpha_{n}\right)\left\|x_{n}-q\right\| \\
& =\beta_{n} \rho\left\|x_{n}-q\right\|+\beta_{n}\|f(q)-q\|+\left(1-\beta_{n}\right) \alpha_{n}\left\|x_{n}-q\right\|+\left(1-\beta_{n}\right) \alpha_{n}\|V q-q\| \\
& +\left(1-\beta_{n}\right) \alpha_{n} \lambda_{n}\|V A q\|+\left(1-\beta_{n}\right)\left(1-\alpha_{n}\right)\left\|x_{n}-q\right\| \\
& \leq \beta_{n} \rho\left\|x_{n}-q\right\|+\beta_{n}\|f(q)-q\|+\left(1-\beta_{n}\right)\left\|x_{n}-q\right\|+\alpha_{n}\|V q-q\|+\alpha_{n} \lambda_{n}\|V A q\| \\
& \leq\left[1-(1-\rho) \beta_{n}\right]\left\|x_{n}-q\right\|+\beta_{n}(\|f(q)-q\|+\gamma\|V q-q\|+\gamma\|V A q\|) .
\end{aligned}
$$

By induction, it follows that

$$
\left\|x_{n}-q\right\| \leq \max \left\{\left\|x_{0}-q\right\|, \frac{1}{1-\rho}(\|f(q)-q\|+\gamma\|V q-q\|+\gamma\|V A q\|)\right\}, \quad \forall n \geq 0 .
$$

Therefore $\left\{x_{n}\right\}$ is bounded and so are $\left\{u_{n}\right\},\left\{A x_{n}\right\},\left\{V x_{n}\right\}$, and $\left\{f\left(x_{n}\right)\right\}$.

Step 2. We claim that $\lim _{n \rightarrow \infty}|| x_{n+1}-x_{n} \|=0$. Setting $y_{n}=\left(I-\lambda_{n} A\right) x_{n}$, since $I-\lambda_{n} A$ be nonexpansive, we have

$$
\begin{aligned}
\left\|y_{n}-y_{n-1}\right\| & =\left\|\left(I-\lambda_{n} A\right) x_{n}-\left(I-\lambda_{n-1} A\right) x_{n-1}\right\| \\
& \leq\left\|\left(I-\lambda_{n} A\right) x_{n}-\left(I-\lambda_{n} A\right) x_{n-1}\right\| \\
& +\left\|\left(I-\lambda_{n} A\right) x_{n-1}-\left(I-\lambda_{n-1} A\right) x_{n-1}\right\| \\
& \leq\left\|x_{n}-x_{n-1}\right\|+\left|\lambda_{n}-\lambda_{n-1}\right|\left\|A x_{n-1}\right\| \\
& \leq\left\|x_{n}-x_{n-1}\right\|+M_{1}\left|\lambda_{n}-\lambda_{n-1}\right|
\end{aligned}
$$

where $\quad M_{1}=\sup \left\{\left\|A x_{n}\right\|: n \in \mathbb{N}\right\}$. On the other hand, from $u_{n-1}=T_{r_{n-1}}\left(x_{n-1}-r_{n-1} B x_{n-1}\right)$ and $u_{n}=T_{r_{n}}\left(x_{n}-r_{n} B x_{n}\right)$, it follows that

$$
F\left(u_{n-1}, y\right)+\left\langle B x_{n-1}, y-u_{n-1}\right\rangle+\varphi(y)-\varphi\left(u_{n-1}\right)+\frac{1}{r_{n-1}}\left\langle y-u_{n-1}, u_{n-1}-x_{n-1}\right\rangle \geq 0, \quad \forall y \in C
$$

and

$$
F\left(u_{n}, y\right)+\left\langle B x_{n}, y-u_{n}\right\rangle+\varphi(y)-\varphi\left(u_{n}\right)+\frac{1}{r_{n}}\left\langle y-u_{n}, u_{n}-x_{n}\right\rangle \geq 0, \quad \forall y \in C .
$$

Substituting $y=u_{n}$ into (3.4) and $y=u_{n-1}$ into (3.5), we have

$$
F\left(u_{n-1}, u_{n}\right)+\left\langle B x_{n-1}, u_{n}-u_{n-1}\right\rangle+\varphi\left(u_{n}\right)-\varphi\left(u_{n-1}\right)+\frac{1}{r_{n-1}}\left\langle u_{n}-u_{n-1}, u_{n-1}-x_{n-1}\right\rangle \geq 0
$$

and

$$
F\left(u_{n}, u_{n-1}\right)+\left\langle B x_{n}, u_{n-1}-u_{n}\right\rangle+\varphi\left(u_{n-1}\right)-\varphi\left(u_{n}\right)+\frac{1}{r_{n}}\left\langle u_{n-1}-u_{n}, u_{n}-x_{n}\right\rangle \geq 0 .
$$

From (A2), we have

$$
\left\langle u_{n}-u_{n-1}, B x_{n-1}-B x_{n}+\frac{u_{n-1}-x_{n-1}}{r_{n-1}}-\frac{u_{n}-x_{n}}{r_{n}}\right\rangle \geq 0,
$$


and then

$$
\left\langle u_{n}-u_{n-1}, r_{n-1}\left(B x_{n-1}-B x_{n}\right)+u_{n-1}-x_{n-1}-\frac{r_{n-1}}{r_{n}}\left(u_{n}-x_{n}\right)\right\rangle \geq 0,
$$

So

$$
\left\langle u_{n}-u_{n-1}, r_{n-1} B x_{n-1}-r_{n-1} B x_{n}+u_{n-1}-u_{n}+u_{n}-x_{n-1}-\frac{r_{n-1}}{r_{n}}\left(u_{n}-x_{n}\right)\right\rangle \geq 0 .
$$

It follows that

$$
\begin{aligned}
& \left\langle u_{n}-u_{n-1},\left(I-r_{n-1} B\right) x_{n}-\left(I-r_{n-1} B\right) x_{n-1}+u_{n-1}-u_{n}+u_{n}-x_{n}-\frac{r_{n-1}}{r_{n}}\left(u_{n}-x_{n}\right)\right\rangle \geq 0, \\
& \left\langle u_{n}-u_{n-1}, u_{n-1}-u_{n}\right\rangle+\left\langle u_{n}-u_{n-1}, x_{n}-x_{n-1}+\left(1-\frac{r_{n-1}}{r_{n}}\right)\left(u_{n}-x_{n}\right)\right\rangle \geq 0 .
\end{aligned}
$$

Without loss of generality, let us assume that there exists a real number $c$ such that $r_{n-1}>\mathrm{c}>0$, for all $n \in \mathbb{N}$. Then, we have

$$
\begin{array}{r}
\left\|u_{n}-u_{n-1}\right\|^{2} \leq\left\langle u_{n}-u_{n-1}, x_{n}-x_{n-1}+\left(1-\frac{r_{n-1}}{r_{n}}\right)\left(u_{n}-x_{n}\right)\right\rangle \\
\leq\left\|u_{n}-u_{n-1}\right\|\left\{\left\|x_{n}-x_{n-1}\right\|+\left|1-\frac{r_{n-1}}{r_{n}}\right|\left\|u_{n}-x_{n}\right\|\right\}
\end{array}
$$

and hence

$$
\begin{gathered}
\left\|u_{n}-u_{n-1}\right\| \leq\left\|x_{n}-x_{n-1}\right\|+\frac{1}{r_{n}}\left|r_{n}-r_{n-1}\right|\left\|u_{n}-x_{n}\right\| \\
\leq\left\|x_{n}-x_{n-1}\right\|+\frac{M_{2}}{c}\left|r_{n}-r_{n-1}\right|
\end{gathered}
$$

where $M_{2}=\sup \left\{\left\|u_{n}-x_{n}\right\|: n \in \mathbb{N}\right\}$. From (3.1), we have

$$
\begin{aligned}
\left\|x_{n+1}-x_{n}\right\|= & \| \beta_{n} f\left(x_{n}\right)+\left(1-\beta_{n}\right)\left[\alpha_{n} V y_{n}+\left(1-\alpha_{n}\right) S u_{n}\right] \\
& -\beta_{n-1} f\left(x_{n-1}\right)-\left(1-\beta_{n-1}\right)\left[\alpha_{n-1} V y_{n-1}+\left(1-\alpha_{n-1}\right) S u_{n-1}\right] \| \\
\leq & \beta_{n} \rho\left\|x_{n}-x_{n-1}\right\|+\left|\beta_{n}-\beta_{n-1}\right|\left\|f\left(x_{n-1}\right)\right\|+\|\left(1-\beta_{n}\right)\left[\alpha_{n} V y_{n}+\left(1-\alpha_{n}\right) S u_{n}\right] \\
& -\left(1-\beta_{n-1}\right)\left[\alpha_{n-1} V y_{n-1}+\left(1-\alpha_{n-1}\right) S u_{n-1}\right] \| \\
= & \beta_{n} \rho\left\|x_{n}-x_{n-1}\right\|+\left|\beta_{n}-\beta_{n-1}\right|\left\|f\left(x_{n}-1\right)\right\| \\
& +\|\left(1-\beta_{n}\right)\left[\alpha_{n} V y_{n}-\alpha_{n} V y_{n-1}+\left(1-\alpha_{n}\right) S u_{n}-\left(1-\alpha_{n}\right) S u_{n-1}\right] \\
& +\left(1-\beta_{n}\right) \alpha_{n} V y_{n-1}-\left(1-\beta_{n-1}\right) \alpha_{n-1} V y_{n-1} \\
& +\left(1-\beta_{n}\right)\left(1-\alpha_{n}\right) S u_{n-1}-\left(1-\beta_{n-1}\right)\left(1-\alpha_{n-1}\right) S u_{n-1} \| \\
\leq & \beta_{n} \rho\left\|x_{n}-x_{n-1}\right\|+\left|\beta_{n}-\beta_{n-1}\right|\left\|f\left(x_{n-1}\right)\right\| \\
& +\left(1-\beta_{n}\right)\left\|\alpha_{n}\left[V y_{n}-V y_{n-1}\right]+\left(1-\alpha_{n}\right)\left[s u_{n}-S u_{n-1}\right]\right\| \\
& +\|\left(\alpha_{n}-\beta_{n} \alpha_{n}-\alpha_{n-1}+\beta_{n-1} \alpha_{n-1}\right) V y_{n-1} \\
& +\left(1-\beta_{n}-\alpha_{n}+\beta_{n} \alpha_{n}-1+\beta_{n-1}+\alpha_{n-1}-\beta_{n-1} \alpha_{n-1}\right) S u_{n-1} \| \\
\leq & \beta_{n} \rho\left\|x_{n}-x_{n-1}\right\|+\left|\beta_{n}-\beta_{n-1}\right|\left\|f\left(x_{n-1}\right)\right\|
\end{aligned}
$$




$$
\begin{aligned}
& +\left(1-\beta_{n}\right) \alpha_{n}\left\|y_{n}-y_{n-1}\right\|+\left(1-\beta_{n}\right)\left(1-\alpha_{n}\right)\left\|u_{n}-u_{n-1}\right\| \\
& +\|\left(\alpha_{n}-\alpha_{n-1}-\beta_{n} \alpha_{n}+\beta_{n} \alpha_{n-1}-\beta_{n} \alpha_{n-1}+\beta_{n-1} \alpha_{n-1}\right) V y_{n-1} \\
& +\left(-\beta_{n}+\beta_{n-1}-\alpha_{n}+\alpha_{n-1}+\beta_{n} \alpha_{n}-\beta_{n} \alpha_{n-1}+\beta_{n} \alpha_{n-1}-\beta_{n-1} \alpha_{n-1}\right) S u_{n-1} \| \\
& \leq \beta_{n} \rho\left\|x_{n}-x_{n-1}\right\|+\left|\beta_{n}-\beta_{n-1}\right|\left\|f\left(x_{n-1}\right)\right\| \\
& +\left(1-\beta_{n}\right) \alpha_{n}\left\{\left\|x_{n}-x_{n-1}\right\|+M_{1}\left|\lambda_{n}-\lambda_{n-1}\right|\right\} \\
& +\left(1-\beta_{n}\right)\left(1-\alpha_{n}\right)\left\{\left\|x_{n}-x_{n-1}\right\|+\frac{M_{2}}{c}\left|r_{n}-r_{n-1}\right|\right\} \\
& +\|\left[\left(\alpha_{n}-\alpha_{n-1}\right)-\beta_{n}\left(\alpha_{n}-\alpha_{n-1}\right)-\left(\beta_{n}-\beta_{n-1}\right) \alpha_{n-1}\right] V y_{n-1} \\
& +\left[-\left(\beta_{n}-\beta_{n-1}\right)-\left(\alpha_{n}-\alpha_{n-1}\right)+\beta_{n}\left(\alpha_{n}-\alpha_{n-1}\right)+\left(\beta_{n}-\beta_{n-1}\right) \alpha_{n-1}\right] S u_{n-1} \| \\
& =\beta_{n} \rho\left\|x_{n}-x_{n-1}\right\|+\left|\beta_{n}-\beta_{n-1}\right|\left\|f\left(x_{n-1}\right)\right\| \\
& +\left(1-\beta_{n}\right) \alpha_{n}\left\|x_{n}-x_{n-1}\right\|+\left(1-\beta_{n}\right) \alpha_{n} M_{1}\left|\lambda_{n}-\lambda_{n-1}\right| \\
& +\left(1-\beta_{n}\right)\left(1-\alpha_{n}\right)\left\|x_{n}-x_{n-1}\right\|+\left(1-\beta_{n}\right)\left(1-\alpha_{n}\right) \frac{M_{2}}{c}\left|r_{n}-r_{n-1}\right| \\
& +\|\left[\left(1-\beta_{n}\right)\left(\alpha_{n}-\alpha_{n-1}\right)-\left(\beta_{n}-\beta_{n-1}\right) \alpha_{n-1}\right] V y_{n-1} \\
& +\left[\left(\beta_{n}-1\right)\left(\alpha_{n}-\alpha_{n-1}\right)+\left(\beta_{n}-\beta_{n-1}\right)\left(\alpha_{n-1}-1\right)\right] S u_{n-1} \| \\
& \leq \beta_{n} \rho\left\|x_{n}-x_{n-1}\right\|+\left(1-\beta_{n}\right)\left\|x_{n}-x_{n-1}\right\| \\
& +\left|\beta_{n}-\beta_{n-1}\right|\left\|f\left(x_{n-1}\right)\right\|+\left(1-\beta_{n}\right) \alpha_{n} M_{1}\left|\lambda_{n}-\lambda_{n-1}\right| \\
& +\left(1-\beta_{n}\right)\left(1-\alpha_{n}\right) \frac{M_{2}}{c}\left|r_{n}-r_{n-1}\right| \\
& +\left(1-\beta_{n}\right)\left(\alpha_{n}-\alpha_{n-1}\right)\left\|V y_{n-1}-S u_{n-1}\right\| \\
& +\left\|\left(\beta_{n}-\beta_{n-1}\right)\left(\alpha_{n-1}-1\right) S u_{n-1}-\left(\beta_{n}-\beta_{n-1}\right) \alpha_{n-1} V y_{n-1}\right\| \\
& +\left(1-\beta_{n}\right) \alpha_{n} M_{1}\left|\lambda_{n}-\lambda_{n-1}\right|+\left(1-\beta_{n}\right)\left(1-\alpha_{n}\right) \frac{M_{2}}{c}\left|r_{n}-r_{n-1}\right| \\
& +\left(1-\beta_{n}\right)\left(\alpha_{n}-\alpha_{n-1}\right)\left\|V y_{n-1}-S u_{n-1}\right\| \\
& +\left|\beta_{n}-\beta_{n-1}\right|\left(\left\|f\left(x_{n-1}\right)\right\|+\alpha_{n-1}\left\|V y_{n-1}\right\|+\left|1-\alpha_{n-1}\right|\left\|S u_{n-1}\right\|\right) \\
& \leq \beta_{n} \rho\left\|x_{n}-x_{n-1}\right\|+\left(1-\beta_{n}\right)\left\|x_{n}-x_{n-1}\right\|+\alpha_{n} M_{1}\left|\lambda_{n}-\lambda_{n-1}\right| \\
& +\frac{M_{2}}{c}\left|r_{n}-r_{n-1}\right|+\left(\alpha_{n}-\alpha_{n-1}\right)\left\|V y_{n-1}-S u_{n-1}\right\| \\
& +\left|\beta_{n}-\beta_{n-1}\right|\left(\left\|f\left(x_{n-1}\right)\right\|+\alpha_{n-1}\left\|V y_{n-1}\right\|+\left|1-\alpha_{n-1}\right|\left\|S u_{n-1}\right\|\right) \\
& \leq\left[1-(1-\rho) \beta_{n}\right]\left\|x_{n}-x_{n-1}\right\|+\alpha_{n} M_{1}\left|\lambda_{n}-\lambda_{n-1}\right|+\frac{M_{2}}{c}\left|r_{n}-r_{n-1}\right| \\
& +\left(\left|\alpha_{n}-\alpha_{n-1}\right|+\left|\beta_{n}-\beta_{n-1}\right|\right) M_{3} \\
& =\left[1-(1-\rho) \beta_{n}\right]\left\|x_{n}-x_{n-1}\right\|+\alpha_{n} M_{1}\left|\lambda_{n}-\lambda_{n-1}\right|+\frac{M_{2}}{c}\left|r_{n}-r_{n-1}\right| \\
& +\left(\frac{\left|\alpha_{n}-\alpha_{n-1}\right|}{\beta_{n}}+\frac{\left|\beta_{n}-\beta_{n-1}\right|}{\beta_{n}}\right) \beta_{n} M_{3} \\
& \leq\left[1-(1-\rho) \beta_{n}\right]\left\|x_{n}-x_{n-1}\right\|+\alpha_{n} M_{1}\left|\lambda_{n}-\lambda_{n-1}\right|+\frac{M_{2}}{c}\left|r_{n}-r_{n-1}\right| \\
& +\left(\frac{\gamma\left|\alpha_{n}-\alpha_{n-1}\right|}{\alpha_{n}}+\frac{\left|\beta_{n}-\beta_{n-1}\right|}{\beta_{n}}\right) \beta_{n} M_{3} \text {, }
\end{aligned}
$$

where $M_{3}=\sup \left\{\max \left\{\left\|V y_{n-1}||,|| S u_{n-1}\right\|, \| f\left(x_{n-1}\right)||\right\}\right\}$. Since conditions (C1)-(C5) by Lemma 2.6, we have $\left\|x_{n+1}-x_{n}\right\| \rightarrow 0$ as $n \rightarrow \infty$.

Step 3. We claim that $\lim _{n \rightarrow \infty}|| x_{n}-S x_{n}||=0$. For each $q \in \Xi$, we note that since $T_{r_{n}}$ is firmly nonexpansive, then we have 


$$
\begin{aligned}
\left\|u_{n}-q\right\|^{2}= & \left\|T_{r_{n}}\left(x_{n}-r_{n} B x_{n}\right)-T_{r_{n}}\left(q-r_{n} B q\right)\right\|^{2} \\
\leq & \left\langle T_{r_{n}}\left(x_{n}-r_{n} B x_{n}\right)-T_{r_{n}}\left(q-r_{n} B q\right), u_{n}-q\right) \\
= & \left\langle\left(x_{n}-r_{n} B x_{n}\right)-\left(q-r_{n} B q\right), u_{n}-q\right\rangle \\
= & \frac{1}{2}\left\{\left\|\left(x_{n}-r_{n} B x_{n}\right)-\left(q-r_{n} B q\right)\right\|^{2}+\left\|u_{n}-q\right\|^{2}\right. \\
& \left.-\left\|\left(x_{n}-r_{n} B x_{n}\right)-\left(q-r_{n} B q\right)-\left(u_{n}-q\right)\right\|^{2}\right\} \\
\leq & \frac{1}{2}\left\{\left\|x_{n}-q\right\|^{2}+\left\|u_{n}-q\right\|^{2}-\left\|x_{n}-u_{n}-r_{n}\left(B x_{n}-B q\right)\right\|^{2}\right\} \\
\leq & \frac{1}{2}\left\{\left\|x_{n}-q\right\|^{2}+\left\|u_{n}-q\right\|^{2}-\left\|x_{n}-u_{n}\right\|^{2}\right. \\
& \left.+2 r_{n}\left\langle x_{n}-u_{n}, B x_{n}-B q\right\rangle-r_{n}^{2}\left\|B x_{n}-B q\right\|^{2}\right\},
\end{aligned}
$$

which imply that

$$
\left\|u_{n}-q\right\|^{2} \leq\left\|x_{n}-q\right\|^{2}-\left\|x_{n}-u_{n}\right\|^{2}+2 r_{n}\left\|x_{n}-u_{n}\right\|\left\|B x_{n}-B q\right\| .
$$

From (3.1) and set $w_{n}:=\alpha_{n} V\left(I-\lambda_{n} A\right) x_{n}+\left(1-\alpha_{n}\right) S u_{n}$, when $y_{n}=\left(I-\lambda_{n} A\right) x_{n}$, then we have

$$
\begin{aligned}
\left\|w_{n}-q\right\|^{2} & =\left\|\alpha_{n} V y_{n}+\left(1-\alpha_{n}\right) S u_{n}-q\right\|^{2} \\
& =\left\|\alpha_{n} V y_{n}+\left(1-\alpha_{n}\right) S u_{n}-\left(1-\alpha_{n}\right) S q+\left(1-\alpha_{n}\right) S q-q\right\|^{2} \\
& =\left\|\alpha_{n}\left(V y_{n}-S q\right)+\left(1-\alpha_{n}\right)\left(S u_{n}-S q\right)+S q-q\right\|^{2} \\
& \leq \alpha_{n}\left\|V y_{n}-S q\right\|^{2}+\left(1-\alpha_{n}\right)\left\|u_{n}-q\right\|^{2} .
\end{aligned}
$$

On the other hand, we note that

$$
\begin{aligned}
\left\|u_{n}-q\right\|^{2} & =\left\|T_{r_{n}}\left(x_{n}-r_{n} B x_{n}\right)-T_{r_{n}}\left(q-r_{n} B q\right)\right\|^{2} \\
& \leq\left\|\left(x_{n}-r_{n} B x_{n}\right)-\left(q-r_{n} B q\right)\right\|^{2} \\
& =\left\|\left(x_{n}-q\right)-r_{n}\left(B x_{n}-B q\right)\right\|^{2} \\
& \leq\left\|x_{n}-q\right\|^{2}-2 r_{n}\left\langle x_{n}-q, B x_{n}-B q\right\rangle+r_{n}^{2}\left\|B x_{n}-B q\right\|^{2} \\
& \leq\left\|x_{n}-q\right\|^{2}-2 r_{n} \beta\left\|B x_{n}-B q\right\|^{2}+r_{n}^{2}\left\|B x_{n}-B q\right\|^{2} .
\end{aligned}
$$

Using (3.8) and (3.9), we note that

$$
\begin{aligned}
\left\|x_{n+1}-q\right\|^{2}= & \left\|\beta_{n} f\left(x_{n}\right)+\left(1-\beta_{n}\right) w_{n}-q\right\|^{2} \\
\leq & \beta_{n}\left\|f\left(x_{n}\right)-q\right\|^{2}+\left(1-\beta_{n}\right)\left\|w_{n}-q\right\|^{2} \\
\leq & \beta_{n} \rho^{2}\left\|x_{n}-q\right\|^{2}+\left(1-\beta_{n}\right)\left\{\alpha_{n}\left\|V y_{n}-S q\right\|^{2}+\left(1-\alpha_{n}\right)\left\|u_{n}-q\right\|^{2}\right\} \\
\leq & \beta_{n} \rho\left\|x_{n}-q\right\|^{2}+\left(1-\beta_{n}\right) \alpha_{n}\left\|V y_{n}-S q\right\|^{2}+\left(1-\beta_{n}\right)\left(1-\alpha_{n}\right)\left\|u_{n}-q\right\|^{2} \\
\leq & \beta_{n} \rho\left\|x_{n}-q\right\|^{2}+\alpha_{n}\left\|V y_{n}-S q\right\|^{2}+\left(1-\beta_{n}\right)\left(1-\alpha_{n}\right) \\
& \times\left\{\left\|x_{n}-q\right\|^{2}-2 r_{n} \beta\left\|B x_{n}-B q\right\|^{2}+r_{n}^{2}\left\|B x_{n}-B q\right\|^{2}\right\} \\
= & \beta_{n} \rho\left\|x_{n}-q\right\|^{2}+\alpha_{n}\left\|V y_{n}-S q\right\|^{2}+\left(1-\beta_{n}\right)\left(1-\alpha_{n}\right) \\
\times & \left\{\left\|x_{n}-q\right\|^{2}-r_{n}\left(r_{n}-2 \beta\right)\left\|B x_{n}-B q\right\|^{2}\right\} \\
= & \beta_{n} \rho\left\|x_{n}-q\right\|^{2}+\alpha_{n}\left\|V y_{n}-S q\right\|^{2}+\left(1-\beta_{n}\right)\left(1-\alpha_{n}\right)\left\|x_{n}-q\right\|^{2} \\
& +\left(1-\beta_{n}\right)\left(1-\alpha_{n}\right) r_{n}\left(r_{n}-2 \beta\right)\left\|B x_{n}-B q\right\|^{2} \\
\leq & \beta_{n} \rho\left\|x_{n}-q\right\|^{2}+\alpha_{n}\left\|V y_{n}-S q\right\|^{2}+\left(1-\beta_{n}\right)\left\|x_{n}-q\right\|^{2} \\
& +\left(1-\beta_{n}\right)\left(1-\alpha_{n}\right) r_{n}\left(r_{n}-2 \beta\right)\left\|B x_{n}-B q\right\|^{2} \\
\leq & {\left[1-(1-\rho) \beta_{n}\right]\left\|x_{n}-q\right\|^{2}+\gamma \beta_{n}\left\|V y_{n}-S q\right\|^{2} } \\
& +\left(1-\beta_{n}\right)\left(1-\alpha_{n}\right) r_{n}\left(r_{n}-2 \beta\right)\left\|B x_{n}-B q\right\|^{2} .
\end{aligned}
$$


Then, we have

$$
\begin{aligned}
\left(1-\beta_{n}\right)\left(1-\alpha_{n}\right) c(2 \beta-d)\left\|B x_{n}-B q\right\|^{2} \leq & \gamma \beta_{n}\left\|V y_{n}-S q\right\|^{2}+\left\|x_{n}-q\right\|^{2}-\left\|x_{n+1}-q\right\|^{2} \\
\leq & \gamma \beta_{n}\left\|V y_{n}-S q\right\|^{2} \\
& +\left\|x_{n}-x_{n+1}\right\|\left(\left\|x_{n}-q\right\|+\left\|x_{n+1}-q\right\|\right) .
\end{aligned}
$$

From $(C 2),\left\{r_{n}\right\} \subset[c, d] \subset(0,2 \beta)$ and $\lim _{n \rightarrow \infty}|| x_{n+1}-x_{n} \|=0$, we obtain

$$
\lim _{n \rightarrow \infty}\left\|B x_{n}-B q\right\|=0 \text {. }
$$

Using (3.7), (3.8) and (3.10), it follows that

$$
\begin{aligned}
\left\|x_{n+1}-q\right\|^{2} \leq & \beta_{n} \rho\left\|x_{n}-q\right\|^{2}+\left(1-\beta_{n}\right) \alpha_{n}\left\|V y_{n}-S q\right\|^{2}+\left(1-\beta_{n}\right)\left(1-\alpha_{n}\right)\left\|u_{n}-q\right\|^{2} \\
\leq & \beta_{n} \rho\left\|x_{n}-q\right\|^{2}+\alpha_{n}\left\|V y_{n}-S q\right\|^{2}+\left(1-\beta_{n}\right)\left(1-\alpha_{n}\right) \\
& \times\left\{\left\|x_{n}-q\right\|^{2}-\left\|x_{n}-u_{n}\right\|^{2}+2 r_{n}\left\|x_{n}-u_{n}\right\|\left\|B x_{n}-B q\right\|\right\} \\
= & \beta_{n} \rho\left\|x_{n}-q\right\|^{2}+\alpha_{n}\left\|V y_{n}-S q\right\|^{2}+\left(1-\beta_{n}\right)\left(1-\alpha_{n}\right)\left\|x_{n}-q\right\|^{2} \\
& -\left(1-\beta_{n}\right)\left(1-\alpha_{n}\right)\left\|x_{n}-u_{n}\right\|^{2}+2\left(1-\beta_{n}\right)\left(1-\alpha_{n}\right) r_{n}\left\|x_{n}-u_{n}\right\|\left\|B x_{n}-B q\right\| \\
\leq & \beta_{n} \rho\left\|x_{n}-q\right\|^{2}+\alpha_{n}\left\|V y_{n}-S q\right\|^{2}+\left(1-\beta_{n}\right)\left\|x_{n}-q\right\|^{2} \\
& -\left(1-\beta_{n}\right)\left(1-\alpha_{n}\right)\left\|x_{n}-u_{n}\right\|^{2}+2 r_{n}\left\|x_{n}-u_{n}\right\|\left\|B x_{n}-B q\right\| \\
\leq & {\left[1-(1-\rho) \beta_{n}\right]\left\|x_{n}-q\right\|^{2}+\gamma \beta_{n}\left\|V y_{n}-S q\right\|^{2} } \\
& -\left(1-\beta_{n}\right)\left(1-\alpha_{n}\right)\left\|x_{n}-u_{n}\right\|^{2}+2 r_{n}\left\|x_{n}-u_{n}\right\|\left\|B x_{n}-B q\right\| .
\end{aligned}
$$

Then, we have

$$
\begin{aligned}
\left(1-\beta_{n}\right)\left(1-\alpha_{n}\right)\left\|x_{n}-u_{n}\right\|^{2} \leq & \left\|x_{n}-q\right\|^{2}-\left\|x_{n+1}-q\right\|^{2}+\gamma \beta_{n}\left\|V y_{n}-S q\right\|^{2} \\
& +2 r_{n}\left\|x_{n}-u_{n}\right\|\left\|B x_{n}-B_{q}\right\| \\
\leq & \left\|x_{n}-x_{n+1}\right\|\left(\left\|x_{n}-q\right\|+\left\|x_{n+1}-q\right\|\right)+\gamma \beta_{n}\left\|V y_{n}-S q\right\|^{2} \\
& +2 r_{n}\left\|x_{n}-u_{n}\right\|\left\|B x_{n}-B q\right\| .
\end{aligned}
$$

From (C1), (C2), (3.13) and $\lim _{n \rightarrow \infty}|| x_{n+1}-x_{n}||=0$, we obtain

$$
\lim _{n \rightarrow \infty}\left\|x_{n}-u_{n}\right\|=0 .
$$

By (C5), we obtain

$$
\lim _{n \rightarrow \infty}\left\|\frac{x_{n}-u_{n}}{r_{n}}\right\|=\lim _{n \rightarrow \infty} \frac{1}{r_{n}}\left\|x_{n}-u_{n}\right\|=0 .
$$

From (3.1), it follows that

$$
\begin{aligned}
\left\|x_{n+1}-S u_{n}\right\| & =\left\|\beta_{n} f\left(x_{n}\right)+\left(1-\beta_{n}\right)\left[\alpha_{n} V y_{n}+\left(1-\alpha_{n}\right) S u_{n}\right]-S u_{n}\right\| \\
& =\left\|\beta_{n} f\left(x_{n}\right)+\left(1-\beta_{n}\right) \alpha_{n} V y_{n}+\left(1-\beta_{n}\right)\left(1-\alpha_{n}\right) S u_{n}-S u_{n}\right\| \\
& =\left\|\beta_{n} f\left(x_{n}\right)+\left(1-\beta_{n}\right) \alpha_{n} V y_{n}+\left(1-\beta_{n}\right) S u_{n}+\left(1-\beta_{n}\right) \alpha_{n} S u_{n}-S u_{n}\right\| \\
& \leq \beta_{n}\left\|f\left(x_{n}\right)-S u_{n}\right\|+\left(1-\beta_{n}\right) \alpha_{n}\left\|V y_{n}-S u_{n}\right\| .
\end{aligned}
$$

By (C1) and (C2), then we get

$$
\lim _{n \rightarrow \infty}\left\|x_{n+1}-S u_{n}\right\|=0 .
$$

Since

$$
\begin{aligned}
\left\|x_{n}-S x_{n}\right\| & \leq\left\|x_{n}-x_{n+1}\right\|+\left\|x_{n+1}-S u_{n}\right\|+\left\|S u_{n}-S x_{n}\right\| \\
& \leq\left\|x_{n}-x_{n+1}\right\|+\left\|x_{n+1}-S u_{n}\right\|+\left\|u_{n}-x_{n}\right\| .
\end{aligned}
$$


By $\lim _{n \rightarrow \infty}|| x_{n+1}-x_{n}||=0$, (3.13) and (3.16), so we obtain

$$
\lim _{n \rightarrow \infty}\left\|x_{n}-S x_{n}\right\|=0
$$

Step 4. Next, we will show that

$$
\limsup _{n \rightarrow \infty}\left\langle(I-f) x^{*}, x_{n}-x^{*}\right\rangle \leq 0 .
$$

Indeed, we choose a subsequence $\left\{x_{n_{i}}\right\}$ of $\left\{x_{n}\right\}$ such that

$$
\limsup _{n \rightarrow \infty}\left\langle(I-f) x^{*}, x_{n}-x^{*}\right\rangle=\limsup _{n \rightarrow \infty}\left\langle(I-f) x^{*}, x_{n_{i}}-x^{*}\right\rangle .
$$

Since ?82? is bounded, there exists a subsequence $\left\{x_{n_{j}}\right\}$ of ?82? which converge weakly to $z \in C$. Without loss of generality, we can assume that $x_{n_{i}} \rightarrow z$. From $\| x_{n}-$ $S x_{n} \| \rightarrow 0$, we obtain $S x_{n_{i}} \rightarrow z$. Now, we will show that $z \in \Xi:=F(S) \cap \operatorname{GMEP}(F, \phi$, $B)$. Let us show $z \in F(S)$. Assume that $z \notin F(S)$. Since ?85? and $S z \neq z$. By the Opial's condition, we obtain

$$
\begin{aligned}
\liminf _{i \rightarrow \infty}\left\|x_{n_{i}}-z\right\| & <\liminf _{i \rightarrow \infty}\left\|x_{n_{i}}-S z\right\| \\
& =\liminf _{i \rightarrow \infty}\left\|x_{n_{i}}-S x_{n_{i}}+S x_{n_{i}}-S z\right\| \\
& \leq \liminf _{i \rightarrow \infty}\left(\left\|x_{n_{i}}-S x_{n_{i}}\right\|+\left\|S x_{n_{i}}-S z\right\|\right) \\
& =\liminf _{i \rightarrow \infty}\left\|S x_{n_{i}}-S z\right\| \\
& \leq \liminf _{i \rightarrow \infty}\left\|x_{n_{i}}-z\right\| .
\end{aligned}
$$

This is a contradiction. Thus, we have $z \in F(S)$.

Next, we will show that $z \in \operatorname{GMEP}(F, \phi, B)$. Since $u_{n}=T_{r_{n}}\left(x_{n}-r_{n} B x_{n}\right)$, we have

$$
F\left(u_{n}, y\right)+\left\langle B x_{n}, y-u_{n}\right\rangle+\varphi(y)-\varphi\left(u_{n}\right)+\frac{1}{r_{n}}\left\langle y-u_{n}, u_{n}-x_{n}\right\rangle \geq 0, \quad \forall y \in C .
$$

From (A2), we also have

$$
\left\langle B x_{n}, y-u_{n}\right\rangle+\varphi(y)-\varphi\left(u_{n}\right)+\frac{1}{r_{n}}\left\langle y-u_{n}, u_{n}-x_{n}\right\rangle \geq F\left(y, u_{n}\right), \quad \forall y \in C .
$$

and hence

$$
\left\langle B x_{n_{i}}, y-u_{n_{i}}\right\rangle+\varphi(y)-\varphi\left(u_{n_{i}}\right)+\left\langle y-u_{n_{i}}, \frac{u_{n_{i}}-x_{n_{i}}}{r_{n_{i}}}\right\rangle \geq F\left(y, u_{n_{i}}\right), \quad \forall y \in C .
$$

For $t$ with $0<t \leq 1$ and $y \in C$, let $y_{t}=t y+(1-t) z$. Since $y \in C$ and $z \in C$, we have $y_{t} \in$ C. So, from (3.19), we have

$$
\begin{aligned}
\left\langle y_{t}-u_{n_{i}}, B y_{t}\right\rangle \geq & \left\langle y_{t}-u_{n_{i}}, B y_{t}\right\rangle-\varphi\left(y_{t}\right)+\varphi\left(u_{n_{i}}\right)-\left\langle y_{t}-u_{n_{i}}, B x_{n_{i}}\right\rangle-\left\langle y_{t}-u_{n_{i}}, \frac{u_{n_{i}}-x_{n_{i}}}{r_{n_{i}}}\right\rangle \\
& +F\left(y_{t}, u_{n_{i}}\right) \\
= & \left\langle y_{t}-u_{n_{i}}, B y_{t}-B u_{n_{i}}\right\rangle+\left\langle y_{t}-u_{n_{i}}, B u_{n_{i}}-B x_{n_{i}}\right\rangle-\varphi\left(y_{t}\right)+\varphi\left(u_{n_{i}}\right) \\
& -\left\langle y_{t}-u_{n_{i}}, \frac{u_{n_{i}}-x_{n_{i}}}{r_{n_{i}}}\right\rangle+F\left(y_{t}, u_{n_{i}}\right) .
\end{aligned}
$$


Since $\left\|u_{n_{i}}-x_{n_{i}}\right\| \rightarrow 0$, we have $\left\|B u_{n_{i}}-B x_{n_{i}}\right\| \rightarrow 0$. Further, from the inverse strongly monotonicity of $B$, we have $\left\langle y_{t}-u_{n_{i}}, B y_{t}-B u_{n_{i}}\right\rangle \geq 0$. So, from (A4), (A5), and the weak lower semicontinuity of $\varphi, \frac{u_{n_{i}}-x_{n_{i}}}{r_{n_{i}}} \rightarrow 0$ and $u_{n_{i}} \rightarrow z$, we have at the limit

$$
\left\langle y_{t}-z, B y_{t}\right\rangle \geq-\varphi\left(y_{t}\right)+\varphi(z)+F\left(y_{t}, z\right)
$$

as $i \rightarrow \infty$. From (A1), (A4), and (3.20), we also get

$$
\begin{aligned}
0 & =F\left(y_{t}, y_{t}\right)+\varphi\left(y_{t}\right)-\varphi\left(y_{t}\right) \\
& \leq t F\left(y_{t}, y\right)+(1-t) F\left(y_{t}, z\right)+t \varphi(y)-(1-t) \varphi(z)-\varphi\left(y_{t}\right) \\
& =t\left[F\left(y_{t}, y\right)+\varphi(y)-\varphi\left(y_{t}\right)\right]+(1-t)\left[F\left(y_{t}, z\right)+\varphi(z)-\varphi\left(y_{t}\right)\right] \\
& \leq t\left[F\left(y_{t}, y\right)+\varphi(y)-\varphi\left(y_{t}\right)\right]+(1-t)\left\langle y_{t}-z, B y_{t}\right\rangle \\
& =t\left[F\left(y_{t}, y\right)+\varphi(y)-\varphi\left(y_{t}\right)\right]+(1-t) t\left\langle y-z, B y_{t}\right\rangle, \\
0 & \leq F\left(y_{t}, y\right)+\varphi(y)-\varphi\left(y_{t}\right)+(1-t)\left\langle y-z, B y_{t}\right\rangle .
\end{aligned}
$$

Letting $t \rightarrow 0$, we have, for each $y \in C$,

$$
F(z, y)+\varphi(y)-\varphi(z)+\langle y-z, B z\rangle \geq 0
$$

This implies that $z \in \operatorname{GMEP}(F, \phi, B)$. Therefore $x^{*} \in \Xi$. It is easy to see that $\operatorname{Pr}_{\curlyvee}(I-$ $f)\left(x^{*}\right)$ is a contraction of $H$ into itself. Hence $H$ is complete, there exists a unique fixed point $x^{*} \in H$, such that $x^{*}=P_{\curlyvee}(I-f)\left(x^{*}\right)$. Since $x^{*}=P_{\curlyvee}(I-f)\left(x^{*}\right)$, we have

$$
\begin{aligned}
\limsup _{n \rightarrow \infty}\left\langle(I-f) x^{*}, x_{n}-x^{*}\right\rangle & =\limsup _{n \rightarrow \infty}\left\langle(I-f) x^{*}, S x_{n}-x^{*}\right\rangle \\
& =\limsup _{n \rightarrow \infty}\left\langle(I-f) x^{*}, S x_{n_{i}}-x^{*}\right\rangle \\
& =\left\langle(I-f) x^{*}, z-x^{*}\right\rangle \leq 0 .
\end{aligned}
$$

Step 5. Last, we will prove $x_{n} \rightarrow x^{*} \in \Upsilon$. It follows from (3.1) that, we compute

$$
\begin{aligned}
\left\|x_{n+1}-x^{*}\right\|^{2}= & \left\|\beta_{n} f\left(x_{n}\right)+\left(1-\beta_{n}\right)\left[\alpha_{n} V\left(I-\lambda_{n} A\right) x_{n}+\left(1-\alpha_{n}\right) S T_{r_{n}}\left(x_{n}-r_{n} B x_{n}\right)\right]-x^{*}\right\|^{2} \\
= & \| \beta_{n}\left[f\left(x_{n}\right)-f\left(x^{*}\right)\right]+\left(1-\beta_{n}\right)\left\{\alpha_{n}\left[V\left(I-\lambda_{n} A\right) x_{n}-V\left(I-\lambda_{n} A\right) x^{*}\right]\right. \\
& \left.+\left(1-\alpha_{n}\right)\left[S T_{r_{n}}\left(x_{n}-r_{n} B x_{n}\right)-x^{*}\right]\right\}+\beta_{n}\left[f\left(x^{*}\right)-x^{*}\right] \\
& +\left(1-\beta_{n}\right) \alpha_{n}\left[V\left(I-\lambda_{n} A\right) x^{*}-x^{*}\right] \|^{2} \\
\leq & \| \beta_{n}\left[f\left(x_{n}\right)-f\left(x^{*}\right)\right]+\left(1-\beta_{n}\right)\left\{\alpha_{n}\left[V\left(I-\lambda_{n} A\right) x_{n}-V\left(I-\lambda_{n} A\right) x^{*}\right]\right. \\
& \left.+\left(1-\alpha_{n}\right)\left[S T_{r_{n}}\left(x_{n}-r_{n} B x_{n}\right)-x^{*}\right]\right\} \|^{2} \\
& +2\left\langle\beta_{n}\left[f\left(x^{*}\right)-x^{*}\right]+\left(1-\beta_{n}\right) \alpha_{n}\left[V\left(I-\lambda_{n} A\right) x^{*}-x^{*}\right], x_{n+1}-x^{*}\right\rangle \\
\leq & \beta_{n}\left\|f\left(x_{n}\right)-f\left(x^{*}\right)\right\|^{2}+\left(1-\beta_{n}\right) \| \alpha_{n}\left[V\left(I-\lambda_{n} A\right) x_{n}-V\left(I-\lambda_{n} A\right) x^{*}\right] \\
& +\left(1-\alpha_{n}\right)\left[S T_{r_{n}}\left(x_{n}-r_{n} B x_{n}\right)-x^{*}\right] \|^{2} \\
& +2 \beta_{n}\left\langle f\left(x^{*}\right)-x^{*}, x_{n+1}-x^{*}\right\rangle+2\left(1-\beta_{n}\right) \alpha_{n}\left\langle V\left(I-\lambda_{n} A\right) x^{*}-x^{*}, x_{n+1}-x^{*}\right\rangle \\
\leq & \beta_{n} \rho^{2}\left\|x_{n}-x^{*}\right\|^{2}+\left(1-\beta_{n}\right)\left(\alpha_{n}\left\|\left(I-\lambda_{n} A\right) x_{n}-\left(I-\lambda_{n} A\right) x^{*}\right\|\right. \\
& \left.+\left(1-\alpha_{n}\right)\left\|T_{r_{n}}\left(x_{n}-r_{n} B x_{n}\right)-x^{*}\right\|\right)^{2} \\
& +2 \beta_{n}\left\langle f\left(x^{*}\right)-x^{*}, x_{n+1}-x^{*}\right\rangle+2\left(1-\beta_{n}\right) \alpha_{n}\left\langle V x^{*}-x^{*}, x_{n+1}-x^{*}\right\rangle \\
& -2\left(1-\beta_{n}\right) \alpha_{n} \lambda_{n}\left\langle V A x^{*}, x_{n+1}-x^{*}\right\rangle \\
\leq & \beta_{n} \rho^{2}\left\|x_{n}-x^{*}\right\|^{2} \\
& +\left(1-\beta_{n}\right)\left(\alpha_{n}\left\|x_{n}-x^{*}\right\|+\left(1-\alpha_{n}\right)\left\|x_{n}-x^{*}\right\|\right)^{2} \\
& +2 \beta_{n}\left\langle f\left(x^{*}\right)-x^{*}, x_{n+1}-x^{*}\right\rangle+2\left(1-\beta_{n}\right) \alpha_{n}\left\langle V x^{*}-x^{*}, x_{n+1}-x^{*}\right\rangle \\
& -2\left(1-\beta_{n}\right) \alpha_{n} \lambda_{n}\left\langle V A x^{*}, x_{n+1}-x^{*}\right\rangle \\
\leq & \beta_{n} \rho^{2}\left\|x_{n}-x^{*}\right\|^{2}+\left(1-\beta_{n}\right)\left\|x_{n}-x^{*}\right\|^{2} \\
& +2 \beta_{n}\left\langle f\left(x^{*}\right)-x^{*}, x_{n+1}-x^{*}\right\rangle+2\left(1-\beta_{n}\right) \alpha_{n}\left\|V x^{*}-x^{*}\right\|\left\|x_{n+1}-x^{*}\right\| \\
= & {\left[1-\left(1-\rho^{2}\right) \beta_{n}\right]\left\|x_{n}-x^{*}\right\|^{2} } \\
& +2 \beta_{n}\left\langle f\left(x^{*}\right)-x^{*}, x_{n+1}-x^{*}\right\rangle+2\left(1-\beta_{n}\right) \gamma \beta_{n}\left\|V x^{*}-x^{*}\right\|\left\|x_{n+1}-x^{*}\right\| . \\
&
\end{aligned}
$$


Setting

$$
\delta_{n}=\frac{1}{1-\rho^{2}}\left\{2\left\langle f\left(x^{*}\right)-x^{*}, x_{n+1}-x^{*}\right\rangle+2\left(1-\beta_{n}\right) \gamma\left\|V x^{*}-x^{*}\right\|\left\|x_{n+1}-x^{*}\right\|\right\} .
$$

By (3.18), the fact that $\lim \sup _{n \rightarrow \infty} \delta_{n} \leq 0$. Therefore, by Lemma 2.6, we conclude that $x_{n} \rightarrow x^{*}$, as $n \rightarrow \infty$. This complete the proof.

Next, the following example shows that all conditions of Theorem 3.1 are satisfied.

Example 3.2. For instance, let $\alpha_{n}=\frac{n+1}{n^{2}+1}, \beta_{n}=\frac{1}{n}, \lambda_{n}=\frac{1}{2(n+1)}$ and $r_{n}=\frac{n}{n+1}$. Then, the sequences $\left\{\alpha_{n}\right\},\left\{\beta_{n}\right\},\left\{\lambda_{n}\right\}$ satisfy the following condition (C1)

$$
\frac{n+1}{n^{2}+1} \cdot \frac{1}{2(n+1)}<\frac{n+1}{n^{2}+1}<\gamma \frac{1}{n} \text {. }
$$

We will show that the condition $(\mathrm{C} 2)$ is achieves. Indeed, we obtain that

$$
\begin{aligned}
& \lim _{n \rightarrow \infty} \beta_{n}=\lim _{n \rightarrow \infty} \frac{1}{n}=0, \\
& \sum_{n=1}^{\infty} \beta_{n}=\sum_{n=1}^{\infty} \frac{1}{n}=\infty,
\end{aligned}
$$

and

$$
\begin{aligned}
\lim _{n \rightarrow \infty} \frac{\beta_{n}-1}{\beta_{n}} & =\lim _{n \rightarrow \infty} \frac{\frac{1}{n-1}}{\frac{1}{n}} \\
& =\lim _{n \rightarrow \infty} \frac{n}{n-1} \\
& =1 .
\end{aligned}
$$

Next, we will show that the condition (C3) is achieves. Indeed, we have that

$$
\begin{aligned}
\lim _{n \rightarrow \infty} \frac{\alpha_{n}-1}{\alpha_{n}} & =\lim _{n \rightarrow \infty} \frac{\frac{(n+1)-1}{(n-1)^{2}+1}}{\frac{n+1}{n^{2}+1}} \\
& =\lim _{n \rightarrow \infty} \frac{\frac{n}{n^{2}-2 n+1+1}}{\frac{n+1}{n^{2}+1}} \\
& =\lim _{n \rightarrow \infty} \frac{n}{n^{2}-2 n+2} \cdot \frac{n^{2}+1}{n+1} \\
& =1 .
\end{aligned}
$$

Next, we will show that the condition (C4) is achieves. We observe that

$$
\begin{aligned}
\sum_{n=1}^{\infty}\left|\lambda_{n}-\lambda_{n-1}\right| & =\sum_{n=1}^{\infty}\left|\frac{1}{2(n+1)}-\frac{1}{2 n}\right| \\
& =\left|\frac{1}{2.2}-\frac{1}{2.1}\right|+\left|\frac{1}{2.3}-\frac{1}{2.2}\right|+\left|\frac{1}{2.4}-\frac{1}{2.3}\right|+\ldots \\
& =\frac{1}{2} .
\end{aligned}
$$

Then, the sequence $\left\{\lambda_{n}\right\}$ satisfy the condition (C4). 
Finally, we will show that the condition (C5) is achieves. We compute

$$
\begin{aligned}
\sum_{n=1}^{\infty}\left|r_{n}-r_{n-1}\right| & =\sum_{n=1}^{\infty}\left|\frac{n}{n+1}-\frac{n-1}{(n-1)+1}\right| \\
& =\sum_{n=1}^{\infty}\left|\frac{n(n)-(n-1)(n+1)}{(n+1) n}\right| \\
& =\sum_{n=1}^{\infty}\left|\frac{n^{2}-n^{2}+1}{(n+1) n}\right| \\
& =\sum_{n=1}^{\infty}\left|\frac{1}{n(n+1)}\right|
\end{aligned}
$$

and

$$
\liminf _{n \rightarrow \infty} r_{n}=\liminf _{n \rightarrow \infty} \frac{n}{n+1}=1 .
$$

Then, the sequence $\left\{r_{n}\right\}$ satisfy the condition (C5).

Corollary 3.3. Let $H$ be a real Hilbert space, $f: C \rightarrow C$ be a $\rho$-contraction with coefficient $\rho \in[0,1)$ and $S, V: C \rightarrow C$ be two nonexpansive mappings. Let $F$ be a bifunction from $C \times C \rightarrow \mathcal{R}$ satisfying (A1)-(A5) and let $\varphi: C \rightarrow \mathcal{R}$ is convex and lower semicontinuous with either (B1) or (B2). Assume that $F(S) \cap M E P(F, \phi)$ is nonempty. Suppose $\left\{x_{n}\right\}$ is a sequence generated by the following algorithm $x_{0} \in C$ arbitrarily:

$$
x_{n+1}=\beta_{n} f\left(x_{n}\right)+\left(1-\beta_{n}\right)\left[\alpha_{n} V x_{n}+\left(1-\alpha_{n}\right) S T_{r_{n}} x_{n}\right],
$$

where $\left\{\alpha_{n}\right\}$ and $\left\{\beta_{n}\right\} \subset(0,1)$ and $r_{n} \in(0,2 \beta)$ satisfy the conditions $(C 1)-(C 3)$ and (C5). Then $\left\{x_{n}\right\}$ converges strongly to $x^{*} \in F(S) \cap \operatorname{MEP}(F, \phi)$, which is the unique solution of the variational inequality:

$$
\left\langle(I-f) x^{*}, x-x^{*}\right\rangle \geq 0, \quad \forall x \in F(S) \cap \operatorname{MEP}(F, \varphi) .
$$

The solution of (3.23) is denoted by $\Delta$. This algorithm strongly converge to $x^{*} \in \Delta$.

Proof. Putting $A, B \equiv 0$ in Theorem 3.1, we can obtain desired conclusion immediately.

Corollary 3.4. Let $H$ be a real Hilbert space, $f: C \rightarrow C$ be a $\rho$-contraction with coefficient $\rho \in[0,1)$ and $S, V: C \rightarrow C$ be two nonexpansive mappings. Let $A: C \rightarrow C$ be an $\alpha$-inverse-strongly monotone. Assume that $F(S)$ is nonempty. Suppose $\left\{x_{n}\right\}$ is a sequence generated by the following algorithm $x_{0} \in C$ arbitrarily:

$$
x_{n+1}=\beta_{n} f\left(x_{n}\right)+\left(1-\beta_{n}\right)\left[\alpha_{n} V\left(I-\lambda_{n} A\right) x_{n}+\left(1-\alpha_{n}\right) S x_{n}\right],
$$

where $\left\{\alpha_{n}\right\}$ and $\left\{\beta_{n}\right\} \subset(0,1)$ and $\lambda_{n} \in(0,2 \alpha)$ satisfy the conditions (C1)-(C4).

Then $\left\{x_{n}\right\}$ converges strongly to $x^{*} \in F(S)$, which is the unique solution of the variational inequality:

$$
\left\langle(I-f) x^{*}, x-x^{*}\right\rangle \geq 0, \quad \forall x \in F(S) .
$$

The solution of (3.25) is denoted by $\Gamma$. This algorithm strongly converge to $x^{*} \in \Gamma$.

Proof. Putting $B \equiv 0$ and $T_{r_{n}} \equiv I$ in Theorem 3.1, we can obtain desired conclusion immediately.

Corollary 3.5. Let $H$ be a real Hilbert space, $f: C \rightarrow C$ be a $\rho$-contraction with coefficient $\rho \in[0,1)$ and $S, V: C \rightarrow C$ be two nonexpansive mappings. Assume that $F(S) \neq$ $\varnothing$. Suppose $\left\{x_{n}\right\}$ is a sequences generated by the following algorithm $x_{0} \in C$ arbitrarily: 


$$
x_{n+1}=\beta_{n} f\left(x_{n}\right)+\left(1-\beta_{n}\right)\left[\alpha_{n} V x_{n}+\left(1-\alpha_{n}\right) S x_{n}\right],
$$

where $\left\{\alpha_{n}\right\}$ and $\left\{\beta_{n}\right\} \subset(0,1)$ satisfy the conditions (C1)-(C3).

Then $\left\{x_{n}\right\}$ converges strongly to $x^{*} \in F(S)$, which is the unique solution of the variational inequality:

$$
\left\langle(I-f) x^{*}, x-x^{*}\right\rangle \geq 0, \quad \forall x \in F(S) .
$$

The solution of (3.27) is denoted by $\Gamma^{\prime}$. This algorithm strongly converge to $x^{*} \in \Gamma^{\prime}$.

Proof. Putting $A, B \equiv 0$ and $T_{r_{n}} \equiv I$ in Theorem 3.1, we can obtain desired conclusion immediately.

Remark 3.6. Corollary 3.5 generalizes and improves the result of Marino and $\mathrm{Xu}[7$, Theorem 3.1].

\section{Acknowledgements}

The authors would like to thank The National Research Council of Thailand (NRCT 2554) and the Faculty of Science, King Mongkut's University of Technology Thonburi (KMUTT) for financial support. Furthermore, the authors would like to express their thanks to the referees for their helpful comments.

\section{Competing interests}

The authors declare that they have no competing interests.

\section{Authors' contributions}

All authors contributed equally and significantly in writing this article. All authors read and approved the final manuscript.

Received: 29 December 2011 Accepted: 11 April 2012 Published: 11 April 2012

\section{References}

1. Blum, E, Oettli, W: From optimization and variational inequalities to equilibrium problems. Math Student. 63, 123-145 (1994)

2. Hartman, P, Stampacchia, G: On some nonlinear elliptic differential functional equations. Acta Math. 115, 271-310 (1966)

3. Cianciaruso, F, Marino, G, Muglia, L, Yao, Y: On a two-step algorithm for hierarchical fixed point problems and variational inequalities. J Inequal Appl 2009, 13 (2009). Article ID 208692

4. Yao, JC, Chadli, O: Pseudomonotone complementarity problems and variational inequalities. pp. 501-558. Handbook of Generalized Convexity and Monotonicity (2005)

5. Kirk, WA: Fixed point theorem for mappings which do not increase distance. Am Math Monthly. 72, $1004-1006$ (1965)

6. Yao, Y, Liou, Y-C, Chen, C-P: Hierarchical convergence of a double-net algorithm for equilibrium problems and variational inequality problems. Fixed Point Theory Appl 2010, 16 (2010). Article ID 642584

7. Marino, G, Xu, H-K: Explicit hierarchical fixed point approach to variational inequalities. J Optim Theory Appl 149, 61-78 (2011). doi:10.1007/s10957-010-9775-1

8. Jitpeera, T, Kumam, P: Hybrid algorithms for minimization problems over the solutions of generalized mixed equilibrium and variational inclusion problems. Mathematical Problems in Engineering 25 (2011). Article ID 648617 doi:10.1155/ 2011/648617

9. Browder, FE: Nonlinear operators and nonlinear equations of evolution in Banach spaces. Proc Symp Pure Math. 18, 78-81 (1976)

10. Peng, JW, Yao, JC: A new hybrid-extragradient method for generalized mixed equilibrium problems and fixed point problems and variational inequality problems. Taiwanese J Math. 12(6):1401-1432 (2008)

11. Xu, HK: Iterative algorithms for nonlinear operators. J Lond Math Soc. 66, 240-256 (2002)

doi:10.1186/1029-242X-2012-82

Cite this article as: Jitpeera and Kumam: A new explicit triple hierarchical problem over the set of fixed points and generalized mixed equilibrium problems. Journal of Inequalities and Applications 2012 2012:82. 